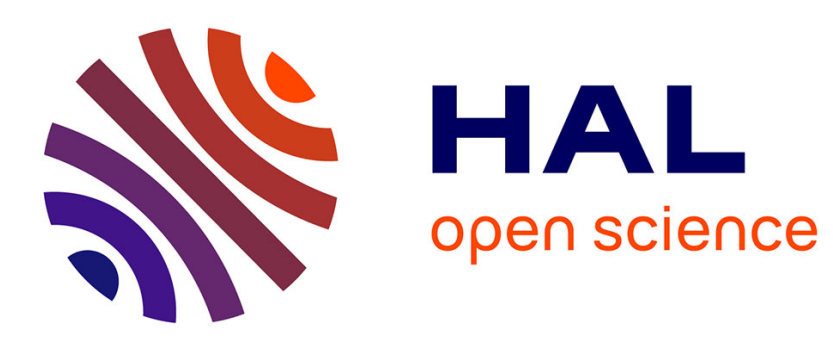

\title{
The Small Outdoor Rover MERLIN and its Assistance System for Tele-Operations
}

Daniel Eck, Manuel Stahl, Klaus Schilling

\section{To cite this version:}

Daniel Eck, Manuel Stahl, Klaus Schilling. The Small Outdoor Rover MERLIN and its Assistance System for Tele-Operations. 6th International Conference on Field and Service Robotics - FSR 2007, Jul 2007, Chamonix, France. inria-00272947

\section{HAL Id: inria-00272947 https://hal.inria.fr/inria-00272947}

Submitted on 14 Apr 2008

HAL is a multi-disciplinary open access archive for the deposit and dissemination of scientific research documents, whether they are published or not. The documents may come from teaching and research institutions in France or abroad, or from public or private research centers.
L'archive ouverte pluridisciplinaire HAL, est destinée au dépôt et à la diffusion de documents scientifiques de niveau recherche, publiés ou non, émanant des établissements d'enseignement et de recherche français ou étrangers, des laboratoires publics ou privés. 


\title{
The Small Outdoor Rover MERLIN and its Assistance System for Tele-Operations
}

\author{
Daniel Eck ${ }^{1}$, Manuel Stahl ${ }^{1}$ and Klaus Schilling ${ }^{1,2}$ \\ 1 Steinbeis Transferzentrum ARS, Würzburg \\ 2 University Würzburg, Informatik VII: Robotics and Telematics, Am Hubland, \\ D-97074 Würzburg, Germany schi@informatik.uni-wuerzburg.de
}

Summary. Tele-operated rovers offer huge application potential in the areas of emergency support, surveillance and security, in particular if they are small and easy to transport. In this context, the MERLIN outdoor rovers have been designed for robust operations in harsh outdoor environments at a total mass below $20 \mathrm{~kg}$. The MERLIN rover system design aspects are described, including the rover on-board data processing and the sensor configuration. Special emphasis is on the remote operations assistance system, composed of the human-machine interface for appropriate presentation of relevant data at the tele-operator workplace and the autonomous reaction capabilities coordinated by the on-board data handling system.

\section{Introduction}

Emergencies and harsh environments often demand dangerous operations, where human personal is to be replaced as far as possible by robots $[1,2,3]$. While in the 90ies the emphasis was on large vehicles, carrying sophisticated sensors for autonomous operations, currently the emphasis is rather towards smaller tele-operated vehicles, enabling better access to narrow work environments. In a broad rover application range including space exploration [4], search and rescue [2] and surveillance, this tendency can be observed. Modern miniaturisation techniques in electronics support this trend without reduction in capabilities, in particular for on-board data processing and control systems, as well as for sensorics [5].

The MERLIN rovers (Mobile Experimental Rover for Locomotion and Intelligent Navigation) were developed in the 90ies, as sensor test vehicles for the European Mars rover design effort MIDD by the Steinbeis center ARS [6]. A new element in this rover family is the Outdoor MERLIN in the mass range between 15 and $20 \mathrm{~kg}$. Therefore, this contribution addresses the system design of small agile mobile robots for tele-operations.

In the following chapter the rover system design aspects are addressed with emphasis on the on-board data processing and sensors. The third chapter 
deals with the implemented tele-operation approaches, including autonomous control functionalities. Experiences from tests to characterize the rover performance are summarized.

\section{System Design of the MERLIN Rover}

The design requirements relate to a payload mass of about $5 \mathrm{~kg}$ at a total rover mass of max $20 \mathrm{~kg}$, a maximum velocity of $40 \mathrm{~km} / \mathrm{h}$ (on road), an operational period of 1 hour, and robust performance in harsh outdoor environments.

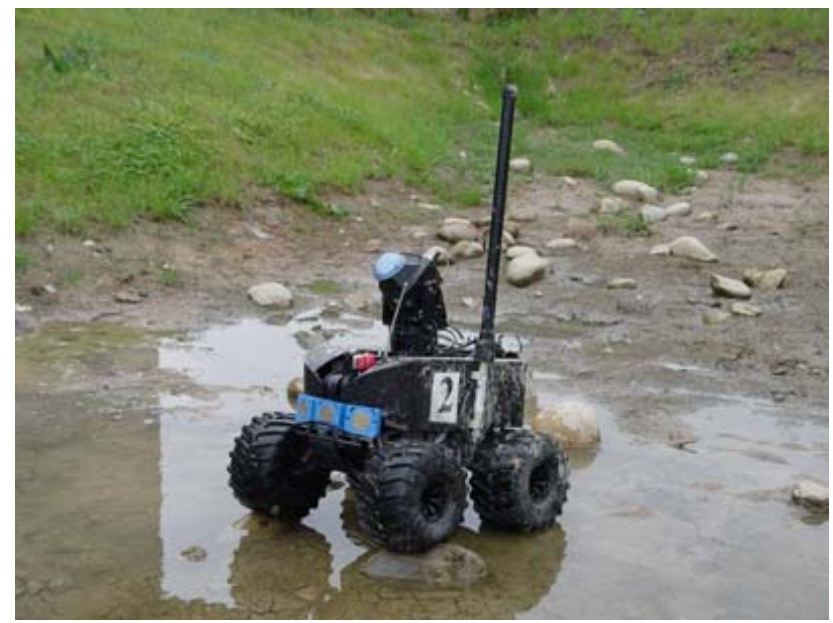

Fig. 1. The Outdoor MERLIN in drive configuration during the test at the European Land Robotics trial ELROB 2005.

To comply with these requirements the MERLIN platform concept has been modified, whereby a new chassis was developed. Furthermore all subsystems of the MERLIN platform concept have been adapted to the new chassis and a new robot, the Outdoor MERLIN, was developed. The Outdoor MERLIN system is composed of four main parts, the mobile robot " Outdoor MERLIN"', the control station, the radio link and the payload, described in more detail in this section.

\subsection{Payload}

The MERLIN vehicle provides a standardized payload interface system, enabling the quick installation of alternative payloads. The payload interface options include by example a grab arm, a surveillance mechanism or a mine search mechanism. Payloads can be changed fast, in order to adapt the robot 
to the execution of different tasks. The mounting slot providing the mechanical interface is placed in the central axis of the vehicle to keep the center of mass in a well defined area. The control of the different payloads is realized via a CAN bus. The modular software concept allows quick integration of new payloads. A regulated power supply of $12 \mathrm{~V}$ is implemented in two independent lines for electronics and for actuators.

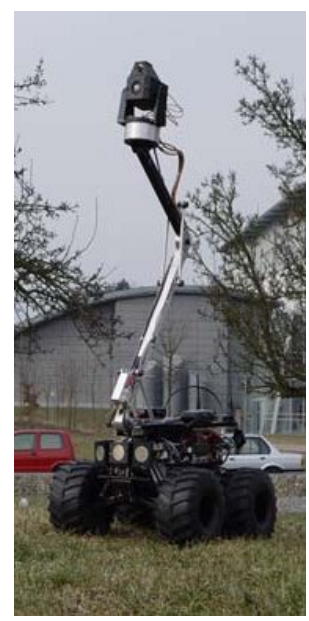

The standard payload configuration is a camera on top of a robotic arm used as mast. The arm can be moved up to approximately $100 \mathrm{~cm}$ elevation. The head is able to change yaw and pitch orientation. It carries two cameras: a surveillance camera with high zoom capabilities and a drive camera with a wide angle objective. So the operator is able to identify objects in the area around the robot, which can be zoomed in for detailed observation. When the operator drives the MERLIN, he uses the drive camera, looking at the environment in front of the MERLIN, including the front wheels, too. Thus the distances to the obstacles can be easily identified, enabling better and faster reactions. Alternative configurations payloads on the mast include also infrared cameras for use during night time.

Fig. 2. The Outdoor MERLIN with the deployed standard mast configuration, elevating the camera for monitoring purposes to $1 \mathrm{~m}$ height for better panoramic view.

\subsection{Control Station}

To tele-operate the MERLIN, a portable control station was developed. The station includes a laptop with joystick, radio devices to send and receive data, the antennas and a charger for the robot's battery packs. All components of the station are stored in a box with the size of $40 \times 60 \times 40 \mathrm{~cm}^{3}$ and a total weight of $21.5 \mathrm{~kg}$. The antenna system consists of a deployable mast and 3 antennas. One bar antenna is for the uplink to the robot. Another bar antenna and a beam antenna are used for the downlink. The Laptop computer hosts the MERLIN Control Software (MRCS) [7] to control the robot and its subsystems. In addition, software for the video and audio treatment is available, too. There is the possibility to operate the control station with a $12 \mathrm{~V}$ battery. Therefore it is very mobile and is not relying on the electricity network. An alternative $220 \mathrm{~V}$ access option is integrated, too.

\subsection{Mobile Vehicle}

For the energy-efficient use in a broad spectrum of environments, a robust vehicle with four wheels was designed. The four wheel drive system can be 
operated with wheels of different diameters. The front axle is steerable. The rover has enough ground clearance to safely pass obstacles of at least 10 $\mathrm{cm}$. Maximum speed is up to $40 \mathrm{~km} / \mathrm{h}$, but for safety reasons the velocity is limited according to path curvature. The low weight below $15 \mathrm{~kg}$ (without payload) supports transportability and enables it to be carried by a single person. It is equipped by an electric motor with a high torque, enabling it to handle significant slopes. The motor provides a power of 1600 watt. The dimensions of the robot are $50 \mathrm{~cm}$ length, $40 \mathrm{~cm}$ width and $60 \mathrm{~cm}$ height (without antenna).

Sensors were chosen to be of maximum robustness and reliability for a broad spectrum of operational environments. Different sensors supply information about the vehicle's status, the position and orientation of the robot, as well as about the environment. Two cameras observe the range before and behind the vehicle. The vehicle is equipped with a basic configuration of

- 4 ultrasonic sensors for obstacle avoidance: 3 looking into drive direction, one placed in the rear.

- 2 infrared distance sensors for obstacle avoidance looking at the right and left side.

- 1 incremental sensor for motor propulsion.

- 1 current sensor for motor current.

- $2 \mathrm{~b} / \mathrm{w}$ cameras on front and rear.

- 1 gyroscope for yaw rate.

- 2 inclinometers for pitch and roll.

- 1 GPS sensor for global position and absolute orientation.

The sensors were extensively tested to verify the suitability for a broad range of typical environments. A particular challenge are electromagnetic cleanliness requirements within the densely packed volume on-board the rover. Intensive shielding of motors to protect from the induced electromagnetic fields are mandatory.

\subsection{Radio Link}

The communication link consists of two components, the uplink from the control station to the vehicle, transports commands from operator, and the downlink in opposite direction, transports the audio and video signal as well as sensor data. Both directions are using a separate radio transmitter. The range of both radio transmitting sets is about 500-1000 meter. The maximum data rate of the uplink is 9600 baud. For transmitting video and audio signal, the downlink has an maximum data rate of $4.9 \mathrm{Mbps}$. The delay of both radio transmitting sets is about 0.5 seconds and depends on the link quality.

For the communication between control station and Outdoor MERLIN a flexible communication protocol is used. It is optimized to save bandwidth and to be open for the integration of new components into the communication protocol. 


\section{Assistance System for Tele-operations}

A human operator is challenged by the remote control of mobile robots in outdoor environments, taking into account environmental characteristics as well as vehicle kinematics and dynamics [8,9]. Moreover, the quality of his work achievable depends on the feedback received from the robot.

Hence, the operator has the need for support to increase mission performance [7]. This support can be provided in two ways:

Passive support the operator is consulted and advised by the system Active support the system intervenes by improving the operator's instructions

Passive assistance is characterized by its consulting nature. In contrast to intervening commands generated by the operator, it provides advice in specific operation scenarios. The quality of the support varies significantly with the quality, quantity and priorities of the information available. Passive support takes place at the Remote Control and is applied through the user interface by visual and audio feedback, which allows the operator to control the robot remotely. Active support is implemented on the robot in the field mainly due to the need for short control loops, which could otherwise be unnecessarily delayed by data transmission via radio link. As defined above, the active support analyzes the operator's commands in relation to the current system status and characteristics of the environment (e.g. current vehicle velocity, threatening obstacles), and adapts his commands to them [7]. In particular, emphasis was placed on implementing autonomous reactions (for obstacle avoidance, " back to base"') and drive assistance systems (for velocity control, maximum centrifugal force) on the robot. The following section focuses on the investigation of active support mechanisms including their classification, organization and realization.

\subsection{User Interface}

The control software is based on a modular concept and is implemented with JAVA as plug-ins for the operating system Eclipse. Therefore, it is easy to integrate control and visualization modules for new hardware components or to adapt the graphic user interface (GUI). Each visualization module has its own window within the application window, which can be moved in an operator defined integrated display area. The operator has the option to save different standard user interfaces for the typical missions. Figure 3 presents an exemplary GUI for the rover tele-operator.

The software supports commercial joysticks or gamepads as input device to control the mobile robot. Often needed functions can be linked with the buttons of these input devices. 


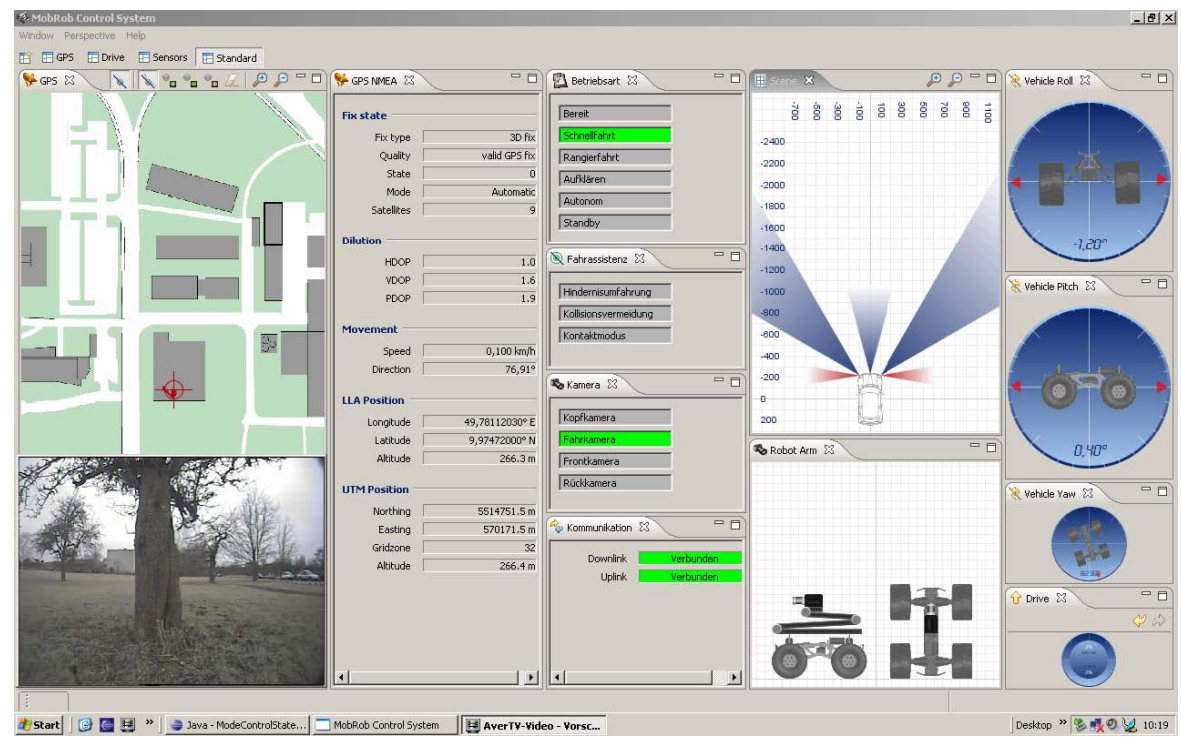

Fig. 3. Typical GUI with integrated windows for navigation, rover status

\subsection{Operation Modes}

There exist several operation modes that can be seen as presets to adapt the robot configuration to the current task.

Ready: This is the initial state after power up. All subsystems are initialized, tested and started. The system waits for user commands. It also indicates that all systems were started successfully.

Fast Driving: The user can drive the vehicle with full speed. Assistance systems can be activated on demand. Payload operation is reduced because the center of mass must not be altered. (i.e. for the camera payload only the head can move but elevation is disabled)

Slow Driving: The maximum speed is limited to provide sensitive steering for maneuvers in narrow places. The payload is active but input is focused on driving.

Surveillance: The vehicle can not be moved. Input mapping is changed to provide better control of the payload. All sensor systems are still active and provide information.

Standby: Energy consumption is reduced to a minimum. Driving is not possible. All sensor systems are still active and provide information to the user.

Autonomy: In autonomy mode the vehicle generates driving commands itself. As soon as the user submits new commands and they are received on the vehicle, the autonomy mode gets disabled and the operation mode 
changes to fast driving. In autonomy mode different behaviors are available that can be activated by the user or automatically.

\begin{tabular}{lllll}
\hline $\begin{array}{l}\text { Operation } \\
\text { Mode }\end{array}$ & Velocity & Sensors & Payload & Joystick \\
\hline Ready & $0 \mathrm{~km} / \mathrm{h}$ & Active & Inactive & Inactive \\
Fast Driving & $12 \mathrm{~km} / \mathrm{h}$ & Active & Limited & Driving / Payload \\
Slow Driving & $4 \mathrm{~km} / \mathrm{h}$ & Active & Limited & Driving / Payload \\
Surveillance & $0 \mathrm{~km} / \mathrm{h}$ & Active & Active & Payload \\
Standby & $0 \mathrm{~km} / \mathrm{h}$ & Active & Inactive & Inactive \\
Autonomy & $10 \mathrm{~km} / \mathrm{h}$ & Active & Inactive & Inactive \\
\hline
\end{tabular}

Table 1. Comparison of operation modes

\subsection{Basic Drive Assistance}

The operator has no permission to disable the features of this level from the autonomous assistance. Nevertheless he can adjust the behavior of each feature by applying situation-dependent presets, which include the needed parameters for the features. This allows for a problem-specific adaptation at this level, but always maintains the minimal support necessary.

It is quite difficult for the operator to assess the current velocity and acceleration of the robot due to delayed data transmission, limited field of vision or inadequate feedback quality. Furthermore, it is nearly impossible for the operator to adjust his commands in real time to variable environmental conditions. For example, it is not possible to maintain the velocity of the vehicle, when the externally applied forces change (downhill force, friction, etc.). Challenges of the implementation are:

- large range of possible velocities

- many different types of environments

- variable robot configuration due to payload changes

The system gets periodic speed commands as an input. Derived from these commands, trajectories are generated in order to modify the current speed towards the desired value, while limiting acceleration forces (throttle, break) avoid overturning of the vehicle. A PID controller calculates the resulting output value, which is delivered to the motor controller in order to achieve the desired velocity of the vehicle.

\subsection{Extended Drive Assistance}

To support the operator during a mission in the "'autonomy mode", the robot must be able to react to obstacles, which are detected on his course. The operator notices obstacles very often retarded or too late, so that he is not able to react fast enough. To avoid this situation, an extended driving 
system was implemented, which processes data from the environment of the vehicle. A key contribution is from ultrasonic and infrared ranging sensors. If the vehicle approaches an obstacle, two autonomous behavior patterns can be chosen: to drive around the obstacle or to push the obstacle.

\subsection{Autonomous Features}

The implementation of partially autonomous functions is necessary. In case the radio link is disturbed, the vehicle must be able to return autonomously until contact to the radio is re-established. Additionally, the operator is able to plan a path for the mission in advance by defining a set of GPS coordinates. The vehicle will try to reach each point avoiding obstacles in its way, even without the communication link. The MERLIN vehicle is able to drive along these paths alone without instructions, while the operator can concentrate on monitoring the area. The autonomous abilities are based on the GPS sensor data, supported by inertial navigation sensors if not enough reference satellites are in the field of view. The accuracy of these GPS coordinates, as well as the quality of the autonomous functions depends on the accuracy of the GPS signals.

\section{Performance Tests}

According to the huge operations range, the robot was subjected to a large number of extensive tests. The robot test area of University Würzburg consists of steep and shallow slopes, many different ground surfaces (asphalt, gravel, grassland, dirt, sand) and various kinds of obstacles (buildings, cars, trees, lampposts, snow piles, tunnels).

Testing took place in a wide range of weather conditions like snowfalls, rains and intense sunshine.

Depending on conditions of the particular test area like roughness, traction and inclination, the configuration of the basic drive assistance and the composition of autonomous reactions were adapted. All tests were accomplished

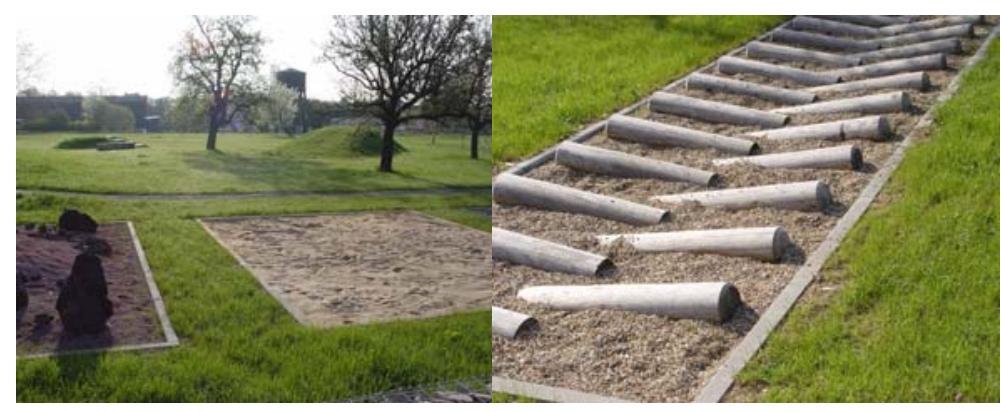

Fig. 4. The rover test area of University Würzburg 
by several operators whose experiences influenced the parameterization and configuration of the drive assistance as well as the overall development process.

In reference tests, the robot was not equipped with any kind of autonomy, not even with the basic drive assistance. The operator could hardly control the acceleration generated by the powerful motor directly, causing frequent turnovers.

Testing the basic drive assistance was characterized by finding parameters for the PID controller in a way that the operator was able to drive safely without noticing the assistance system. This allowed controlling the robot reliably in the operator's field of view.

To emphasize the efficiency of the control algorithm an outdoor test was done driving on grassland and then climbing a hill. The results show that the velocity can be kept in a close region (standard deviation about 6of the desired speed. This is especially necessary when operating with full autonomy.

Controlling the robot outside this area using the small experimental onboard camera led to collisions in many cases due to the highly restrictive field of vision. The faster the robot was driven, the more insufficient was the tele-operations performance of the operator.

The next step was the addition of various sets of autonomous reactions which increased the quality of robot control significantly. Depending on the composition of autonomous features, it was possible to enhance the operator's ability to react in critical situations. For example, navigating the robot through a tree grove in the testing area was possible without colliding with any trees.

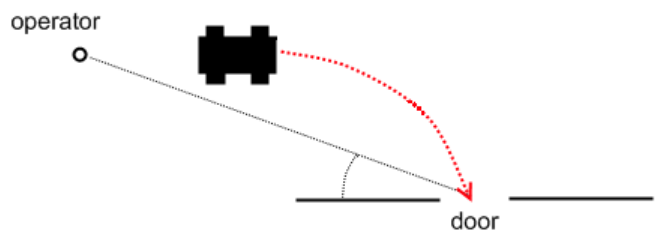

Fig. 5. Obstacle avoidance test setup

\begin{tabular}{l|llll}
\hline Angle & $90^{\circ}$ & $60^{\circ}$ & $30^{\circ}$ & $0^{\circ}$ \\
Improvement & $-20 \%$ & $0 \%$ & $+10 \%$ & $+20 \%$ \\
\hline
\end{tabular}

Table 2. Gain in mission time with obstacle avoidance

Table 2 shows some test results, where the task was to drive through a door as quickly as possible. This was done without and with obstacle avoidance. The view angle of the operator relative to the door was shifted four times. The values give the average improvement of the time it took to drive through the door with the obstacle avoidance compared to the time it took without the assistance.

If the operator has a clear view of what the vehicle is doing, the driving assistance can lead to a slightly worse performance. In this case, the reason is the assistance system breaking the vehicle before passing through the door. 
But as soon as the operator has to guess, where the vehicle is exactly, the assistance system reduces the risk of collisions and also improves the time it takes to complete the mission.

\section{Conclusions}

The Outdoor MERLIN ${ }^{T M}$ vehicles are characterized by compact dimensions at a mass below $20 \mathrm{~kg}$ and robust operations. In order to support save teleoperations, a drive assistant system has been developed based on autonomous functionalities implemented in the on-board microprocessor, as well as by a data interpretation and analysis system implemented in the remote control station. Preliminary tests exhibit a safer, improved performance, when teleoperations is supported by this drive assistance system.

\section{References}

1. Sheridan, T.B.: Telerobotics, automation and human supervisory control. The MIT Press (1992)

2. H. Baier, F. Driewer, K.S.: Robot/human rescue teams: A user requirement analysis. Advanced Robotic 19 (2005) 819-838

3. F. Driewer, K.S.: Remote control of mobile robots for emergencies. Proceedings of the 16th IFAC World Congress (2005) Tu-E15-TO/3

4. Schilling, K.: Control of planetary rovers. special issue of Control Engineering Practice 5 (1997) 823ff

5. Everett, H.R.: Sensors for mobile robots. AK Peters (1995)

6. K. Schilling, Q.M.: The merlin vehicles for outdoor applications. SPIE conference proceedings "Unmanned Ground Vehicle Technology IV" (Orlando 2002) 43-49

7. M. Frank, S. Busch, P.D.: Teleoperations of a mobile outdoor robot with adjustable autonomy. ACIDCA-ICMI Proceedings (2005) O-8

8. R. Siegwart, I.R.N.: Introduction to Autonomous Mobile Robots. Bradford Books (2004)

9. U. Kiencke, L.N.: Automotive Control Systems. Springer Verlag (2000) 